\title{
Excursions in Brownian motion
}

\author{
Kai Lai Chung*
}

\author{
Dedicated to the Memory of Paul Lévy
}

\section{Introduction}

Paul Lévy initiated his profound study of Brownian motion on the line in his article [10] of 1939 and expounded it in one chapter of his book [11]. The article contained a wealth of ideas that inspired a generation of research. A pivot in his approach is the time set when the Brownian path takes the value zero. His idea was to use this set to partition the time axis, so as to resolve the behavior of the path into two parts: the location of the zeros, and the motion in a zero-free interval. This idea is a natural extension of the consideration of successive entrances into a fixed state in a discrete time recurrent Markov chain. But since the zeros of a Brownian path do not form a well-ordered set in the natural order of the line, the execution of the intuitive ideas is not easy. Indeed, Lévy had recourse to another time set, that when the path is surpassing its previous maximum, which he found to be of the same stochastic structure as the zero-set. He based his analysis on the new set, which also led him to the discovery of local time. Despite this brilliant detour, it turned out that a direct attack on the zeros brought quick success, as shown in Theorem 1 below. Moreover, once the crucial calculations have been made, the rest of the denouement follows the pattern of last-exit phenomenon now familiar in Markov processes. The analogy may be pushed further by treating "zero" as a unique boundary point. There is much to be gained from the analogy even from the analytic point of view. For many explicit expressions reveal themselves to be the results of juxtapositions and cancellations of basic probabilistic quantities, and their combinations and transformations are facilitated by the probabilistic insight. This is the gist of the contents of $\S 2$, which may be regarded as a re-stumping of Lévy's old ground with a new guide.

\footnotetext{
* Research supported in part by NSF grant 41710.
} 
The Brownian motion restricted to the maximal zero-free interval containing the time $t$ is called the "excursion process straddling $t$ ", and the portion of it up to $t$ is called the "meandering process ending at $t$ ". The latter term is borrowed from $\mathrm{D}$. L. Iglehart. The basic structure of meandering is given in $\S 3$, that of excursion in $\S 4$. It is a key fact that conditionally on the duration of each process, its law no longer depends on $t$, namely on its location along the time axis. By way of treating a particularly interesting functional, we derive the distribution of the maximum of each process. It is noteworthy that the result for excursion can be obtained from the corresponding distribution for the unrestricted Brownian motion by two successive partial differentiations, followed each time by setting the respective variable equal to zero. This procedure seems to mimic the action of tying down the path to vanish at both ends of the excursion. The resulting distribution may well be new and presents some analytic interest. This is pursued further in $\S 5$. It should be clear that the method of deriving these distributions is applicable to other functionals. We content ourselves with studying occupation times in $\S 6$, and end by extending an assertion by Lévy concerning the second moment of the occupation time of a neighborhood of zero during an excursion. This apparently fills a gap in the literature. Making use of the results in this paper it is now possible to carry further Lévy's approach to local time problems. A note on this will appear elsewhere in joint work with R. T. Durrett. [4b]. In the final $\S 7$ we show how to obtain the clues to Brownian excursions by obvious analogy with the boundary theory for Markov chains.

Some of the results in this paper were announced in [4].

\section{Basic calculations}

Let $B=\{B(t), t \geqq 0\}$ be the standard Brownian motion with all paths continuous. For each $t>0$, we define

$$
\begin{aligned}
& \gamma(t)=\sup \{s \mid s \leqq t ; B(s)=0\} \\
& \beta(t)=\inf \{s \mid s \geqq t ; B(s)=0\}
\end{aligned}
$$

Then $\gamma(t)$ is the last zero of $B$ before $t$, and $\beta(t)$ is the first zero of $B$ after $t$. Since $B(\cdot)$ is continuous, and $P\{B(t)=0\}=0$, we have almost surely

$$
\gamma(t)<t<\beta(t) .
$$

This is true for each $t$, hence also, e.g., for all rational $t$ simultaneously. The stochastic interval $(\gamma(t), \beta(t))$ is called the interval of excursion straddling $t$. Clearly $B$ keeps the same sign in each such interval; let $|B|=Y$, which is known as the reflecting Brownian motion. 
The first entrance time into the singleton $\{x\}$ will be denoted by $T_{x}$,

$$
T_{x}=\inf \{s \mid s>0 ; B(s)=x\} .
$$

Recall from classical theory the following formulas obtained by the reflection principle. For $t>0$ and arbitrary $x$,

for $t>0, x>0, y>0$,

$$
P^{0}\left\{T_{x} \in d t\right\}=\frac{|x|}{\sqrt{2 \pi t^{3}}} e^{-x^{2} / 2 t} d t
$$

$$
P^{x}\left\{B(t) \in d y ; T_{0}>t\right\}=\frac{1}{\sqrt{2 \pi t}}\left\{e^{-(x-y)^{2 / 2 t}}-e^{-(x+y)^{2 / 2 t}}\right\} d y .
$$

Here $P^{x}$ denotes the probability associated with the Brownian motion starting from $x$, and $P^{0}$ will be usually written as $P$. The differential notation above as an abbreviation for the corresponding integrated formula will be used throughout the paper.

It follows that if $0<s<t, x>0, y>0$, then we have from the meaning of $\gamma(t)$ that

$$
\begin{gathered}
P\{\gamma(t) \leqq s ; Y(s) \in d x ; Y(t) \in d y\}= \\
=P\{Y(s) \in d x ; Y(u) \neq 0 \text { for all } u \in[s, t] ; Y(t) \in d y\}= \\
=P\{Y(s) \in d x\} P^{x}\left\{B(t-s) \in d y ; T_{0}>t-s\right\}= \\
=\sqrt{\frac{2}{\pi s}} e^{-x^{2} / 2 s} d x \frac{1}{\sqrt{2 \pi(t-s)}}\left\{e^{\left.-(x-y)^{2 / 2(t-s)}-e^{-(x+y)^{2} / 2(t-s)}\right\} d y .}\right.
\end{gathered}
$$

The first major step is to integrate out $d x$ in the above. Straightforward calculation yields

$$
\int_{0}^{\infty} \exp \left\{-\frac{x^{2}}{2 s}-\frac{(x \pm y)^{2}}{2(t-s)}\right\} d t=\int_{\mp w}^{\infty} e^{-z^{2} / 2} d z e^{-y^{2} / 2 t} \sqrt{\frac{s(t-s)}{t}}
$$

where $w=y \sqrt{\frac{s}{t(t-s)}}$. Using this in (2.3) we obtain

$$
P\{\gamma(t) \leqq s ; Y(t) \in d y\}=\frac{2 d y}{\pi \sqrt{t}} e^{-y^{2} / 2 t} \int_{0}^{w} e^{-z^{2} / 2} d z .
$$

A simple differentiation with respect to $s$ gives the key formula below.

Theorem 1. We have for $0<s<t, y>0$,

$$
P\{\gamma(t) \in d s ; Y(t) \in d y\}=\frac{y}{\pi \sqrt{s(t-s)^{3}}} e^{-y^{2} / 2(t-s)} d s d y .
$$


It is trivial to integrate out dy in the above; we obtain

$$
\begin{gathered}
P\{\gamma(t) \in d s\}=\frac{d s}{\pi \sqrt{s(t-s)}} \\
P\{\gamma(t) \leqq s\}=\frac{2}{\pi} \arcsin \sqrt{s / t}
\end{gathered}
$$

Now Theorem 1 can be cast in the following conditional form.

\section{Corollary.}

$$
P\{Y(t) \in d y \mid \gamma(t)=s\}=\frac{y}{t-s} e^{-y^{2 / 2(t-s)}} d y
$$

The last result corresponds to Lévy's Theorem 42.5 in [11] which played an essential role in his treatment. He stated it in an apparently more general form and proved it in an entirely different way. His method is to consider another process $Y_{1}$ defined by

$$
Y_{1}(t)=\max _{0 \leqq s \leqq t} B(s)-B(t)
$$

and shown to be equivalent in law to $Y$ (see [6; p. 32] for a neat proof of the latter assertion). He then used the joint distribution of $\max _{0 \leqq s \leqq t} B(s)$ and $B(t)$ to derive an analogue of (2.7) for $Y_{1}$. My inability to follow his arguments was the original motivation for the present investigation. The method used here is more direct and without difficulties. In the language of point processes, where the points are the zeros of the Brownian motion, the conditioning in (2.7) is that of a "horizontal window" whereas Lévy's is a "vertical window".* As a matter of fact, since $\gamma(t)$ depends on $t$ and is not an optional random variable, formula $(2.7)$ by itself is not as convenient as its source (2.5).

Integrating (2.5) over $s$ from 0 to $t$, we obtain

$$
\sqrt{\frac{2}{\pi t}} e^{-y^{2} / 2 t}=P\{Y(t) \in d y\} / d y=\int_{0}^{t} \sqrt{\frac{2}{\pi S}} \frac{y}{\sqrt{2 \pi(t-s)^{3}}} e^{-y^{2} / 2(t-s)} d s .
$$

The identity of the first and last members above may be verified analytically, but its importance is due to the probabilistic meaning. The indicated grouping of factors in the integrand is to bring out a fundamental feature of the excursion, namely the last-exit decomposition of the $Y$ process. To explain this and to pursue a remarkable analogy with known results for Markov chains, we introduce the appropriate nota-

\footnotetext{
* Durrett was able to justify Lévy's arguments after considerable labor.
} 
tion below:

$$
\begin{gathered}
p(t ; x, y)=\frac{1}{\sqrt{2 \pi t}} e^{-(x-y)^{2 / 2 t}} ; \\
q(t ; x, y)=p(t ; x, y)-p(t ; x,-y) ; \\
g(t ; 0, y)=\frac{|y|}{\sqrt{2 \pi t^{3}}} e^{-y^{2} / 2 t} .
\end{gathered}
$$

Note $g(t ; 0, y)=\left.(\partial / \partial x) p(t ; x, y)\right|_{x=0}$ for $y>0$. Then (2.8) becomes, after cancelling a factor 2 ,

$$
p(t ; 0, y)=\int_{0}^{t} p(s ; 0,0) g(t-s ; 0, y) d s .
$$

This is the last-exit (from 0) decomposition of which the analogue in Markov chains is

$$
p_{0 j}(t)=\int_{0}^{t} p_{00}(s) g_{0 j}(t-s) d s,
$$

see $[1 ; p .201]$. Now observe that $p(s ; 0,0)=p(s ; y, y)$, so that $(2.10)$ may be rewritten as

$$
p(t ; 0, y)=\int_{0}^{t} g(s ; 0, y) p(t-s ; y, y) d s .
$$

In view of the interpretation in (2.1), this is just the first-entrance (into y) decomposition of which the analogue in Markov chains is

$$
p_{0 j}(t)=\int_{0}^{t} f_{0 j}(s) p_{j j}(t-s) d s,
$$

see $\left[1 ;\right.$ p. 205]. In Markov chains the two functions $f_{0 j}$ and $g_{0 j}$ are of course in general different. Here the fact that the same function $g$ serves in both decompositions is another manifestation of the rich symmetry inherent in the Brownian motion. It tends however to confound matters also. The analogy with Markov chains now enables us to discover and exploit latent relations obscured by explicit expressions. First we realize that $\{g(t ; 0, \cdot), t>0\}$ is an entrance law for the taboo transition semigroup with $\{0\}$ as the taboo set, i.e., for $0<s<t$ and $y>0$, we have

$$
\int_{0}^{\infty} g(s ; 0, x) q(t-s ; x, y) d x=g(t ; 0, y) .
$$

This can again be verified analytically but its probabilistic meaning is clear. Next, initiating the procedure in Markov chains [1; p. 207], we put

$$
\begin{gathered}
g(t ; 0)=\int_{0}^{\infty} g(t, 0, y) d y=\frac{1}{\sqrt{2 \pi t}} \\
h(t ; y)=\frac{g(t ; 0, y)}{g(t ; 0)}=\frac{y}{t} e^{-y^{2} / 2 t}
\end{gathered}
$$


Rewrite (2.4) and (2.6) as follows:

$$
\begin{gathered}
\Gamma(s, t ; d y)=P\{\gamma(t) \leqq s ; Y(t) \in d y\}, \\
\Gamma(s, t)=P\{\gamma(t) \leqq s\} .
\end{gathered}
$$

Then (2.5) and (2.6) become, respectively,

$$
\begin{gathered}
\frac{\partial}{\partial s} \Gamma(s, t ; d y)=2 p(s ; 0,0) g(t-s ; 0, y) d y, \\
\frac{\partial}{\partial s} \Gamma(s, t)=2 p(s ; 0,0) g(t-s ; 0) .
\end{gathered}
$$

The last-exit formula (2.8) may now be written as

$$
P\{Y(t) \in d y\} / d y=\int_{0}^{t} h(t-s ; y) d_{s} \Gamma(s, t)
$$

and becomes a particular case of the more general formula, valid for $0 \leqq s_{1} \leqq t$ :

$$
P\left\{\gamma(t) \leqq s_{1} ; Y(t) \in d y\right\} / d y=\int_{0}^{s_{1}} h(t-s ; y) d_{s} \Gamma(s, t),
$$

which is the full force of (2.5).

We need another quantity to be introduced in the next proposition.

Proposition 2. For $s>0$ and $t>0$, we have

$$
\int_{0}^{\infty} g(s ; 0, x) g(t ; 0, x) d x=\frac{1}{\sqrt{8 \pi(s+t)^{3}}} .
$$

Proof. The left member is equal to

which is easily evaluated.

$$
\int_{0}^{\infty} \frac{1}{2 \pi \sqrt{(s t)^{3}}} x^{2} \exp \left(-\frac{(s+t) x^{2}}{2 s t}\right) d x
$$

The quantity analogous to that in (2.17) for Markov chains is $\sum_{j} g_{0 j}(s) f_{j 0}(t)$. It is introduced in [2; Theorem 6.4] when 0 is regarded as a boundary point, as it might well be also in the present study. Hence we shall use a similar notation below:

$$
\theta(t)=\frac{1}{\sqrt{8 \pi t^{3}}} .
$$

Indeed, we could have obtained $\theta$ from analogy with some fundamental relations in boundary theory, instead of the direct calculation in Proposition 2. This approach is given in $\S 7$ for readers who are acquainted with boundary theory for Markov chains.

To proceed, we multiply (2.5) in the form of (2.14) by the following formula, for $0<t<u$ :

$$
P\{\beta(t) \in d u \mid Y(t)=y\}=P^{y}\left\{T_{0} \in d u-t\right\}=g(u-t ; 0, y) d u
$$


which is obvious by the Markov property of $Y$. The result is

$$
\begin{gathered}
P\{\gamma(t) \in d s ; Y(t) \in d y ; \beta(t) \in d u\}= \\
=2 p(s ; 0,0) g(t-s ; 0, y) g(u-t ; 0, y) d s d y d u .
\end{gathered}
$$

Integrating out $d y$ in the above by using Proposition 2, we obtain the next proposition.

Proposition 3. For $0<s<t<u$, we have

$$
P\{\gamma(t) \in d s ; \beta(t) \in d u\}=\frac{d s d u}{2 \pi \sqrt{s(u-s)^{3}}}=2 p(s ; 0.0) \theta(u-s) d s d u
$$

Let us introduce two more random variables:

$$
L^{-}(t)=t-\gamma(t) ; \quad L(t)=\beta(t)-\gamma(t) .
$$

We call $(\gamma(t), t)$ the interval of meandering ending at $t$. Thus $L^{-}(t)$ is the duration of the meandering ending at $t$, whereas $L(t)$ is the duration of the excursion straddling $t$. For later reference we record (2.6) in the form

$$
P\left\{L^{-}(t) \in d r\right\}=\frac{d r}{\pi \sqrt{r(t-r)}}, \quad 0<r<t ;
$$

from which we obtain

$$
P\left\{L(t) \in d l \mid L^{-}(t)=r\right\}=\frac{1}{2} \sqrt{\frac{r}{l^{3}}} d l, \quad 0<r<t \wedge l .
$$

It is sometimes more convenient to use the pair $(\gamma(t), L(t))$ or $\left(L^{-}(t), L(t)\right)$ rather than $(\gamma(t), \beta(t))$ to identify the interval of excursion straddling $t$, as we shall soon see.

\section{The meandering process and its maximum}

For each $t>0$, the process $Y$ restricted to the interval $(\gamma(t), t)$ will be called the meandering process ending at $t$. Precisely, we define $Z$ as follows:

$$
Z(u)=Y(\gamma(t)+u) \text { for } 0 \leqq u \leqq L^{-}(t) .
$$

For each $t>0$ and $u \geqq 0, Z$ is defined only on the measurable sample set $\left\{u \leqq L^{-}(t)\right\}$. It would be futile to define it by decree elsewhere and we desist from doing so. The joint law of $\gamma(t)$ and the $Z$ process is given by the next theorem.

Theorem 4. Let $m \geqq 1,0<u_{1}<\ldots<u_{m}<t-s<t$, and $y_{1}, \ldots, y_{m+1}$ be arbitrary positive numbers. We have

$$
P\left\{\gamma(t) \in d s ; Z\left(u_{1}\right) \in d y_{1} ; \ldots ; Z\left(u_{m}\right) \in d y_{m} ; Y(t) \in d y_{m+1}\right\}=
$$

$$
\begin{gathered}
=2 p(s ; 0,0) d s g\left(u_{1} ; 0, y_{1}\right) d y_{1} q\left(u_{2}-u_{1} ; y_{1}, y_{2}\right) d y_{2} \ldots q\left(u_{m}-u_{m-1} ; y_{m-1}, y_{m}\right) d y_{m} . \\
\cdot q\left(t-s-u_{m} ; y_{m}, y_{m+1}\right) d y_{m+1} .
\end{gathered}
$$


Remark. When there is no $u$, i.e., when $m=0$, the formula above reduces to (2.5) or (2.14).

Proof. It is sufficient to indicate the proof for $m=2$. Let $\varphi_{1}, \ldots, \varphi_{m+1}$ be bounded continuous functions on $(0, \infty)$; and for fixed $s>0$ let

$$
d_{n k}=\frac{k s}{2^{n}}, \quad 0 \leqq k \leqq 2^{n} ; \quad I_{n k}=\left[d_{n, k-1}, d_{n k}\right), \quad 1 \leqq k \leqq 2^{n}
$$

If $d_{n k}+u_{1}<t$ and $\gamma(t) \in I_{n k}$, then $\gamma(t)=\gamma\left(d_{n k}+u_{1}\right)$; thus $\left\{\gamma(t) \in I_{n k}\right\}=\left\{\gamma\left(d_{n k}+u_{1}\right) \in\right.$ $\left.\in I_{n k} ; \beta\left(d_{n k}+u_{1}\right)>t\right\}$. Hence we have

$$
\begin{gathered}
E\left\{\gamma(t)<s ; \varphi_{1}\left(Z\left(u_{1}\right)\right) \varphi_{2}\left(Z\left(u_{2}\right)\right) \varphi_{3}(Y(t))\right\}= \\
=\lim _{n \rightarrow \infty} \sum_{k=1}^{2^{n}} E\left\{\gamma\left(d_{n k}+u_{1}\right) \in I_{n k} ; \beta\left(d_{n k}+u_{1}\right)>u_{2} ;\right. \\
\left.\varphi_{1}\left(Y\left(d_{n k}+u_{1}\right)\right) \varphi_{2}\left(Y\left(d_{n k}+u_{2}\right)\right) \varphi_{3}(Y(t))\right\} .
\end{gathered}
$$

Here and hereafter we write $E(\Lambda ; \varphi)$ for the expectation of $\varphi$ over the set $\Lambda$. By Theorem 1, followed by Markov property of $Y$ applied at $d_{n k}+u_{1}$, the $k^{\text {th }}$ term above is evaluated as follows:

$$
\begin{gathered}
2 \int_{I_{n k}} p(r ; 0,0) d r \int_{0}^{\infty} g\left(d_{n k}-r+u_{1} ; 0, y\right) \varphi_{1}(y) \cdot \\
\cdot E^{y}\left\{T_{0}>t-d_{n k}-u_{1} ; \varphi_{2}\left(Y\left(u_{2}-u_{1}\right)\right) \varphi_{3}\left(Y\left(t-d_{n k}-u_{1}\right)\right)\right\} d y
\end{gathered}
$$

where $E^{y}$ is the expectation induced by $P^{y}$. For $r \in[0, s)$ let us put

$$
r_{n}=\left(\left[\frac{2^{n} r}{s}\right]+1\right) \frac{s}{2^{n}}
$$

where the square bracket denotes the greatest integer function, so that $r_{n}=d_{n k}$ if $r \in I_{n k}$, for $1 \leqq k \leqq 2^{n}$. Then if we sum (3.3) over $k$, we obtain

$$
\begin{gathered}
2 \int_{0}^{s} p(r ; 0,0) d r \int_{0}^{\infty} g\left(r_{n}-r+u_{1} ; 0, y_{1}\right) \varphi_{1}\left(y_{1}\right) d y_{1} . \\
\cdot \int_{0}^{\infty} q\left(u_{2}-u_{1} ; y_{1}, y_{2}\right) \varphi_{2}\left(y_{2}\right) d y_{2} \int_{0}^{\infty} q\left(t-r_{n}-u_{2} ; y_{2}, y_{3}\right) \varphi_{3}\left(y_{3}\right) d y_{3} .
\end{gathered}
$$

Now $g(u ; 0, y)$ and $q(u ; x, y)$ are continuous in $u>0$ for fixed $y$ and $x$, and for $0<a \leqq$ $\leqq u \leqq b<\infty$ there is the easy domination

$$
g(u ; 0, y) \leqq \frac{|y|}{\sqrt{2 \pi a}} e^{-y^{2} / 2 b} ; \quad q(u ; x, y) \leqq \frac{1}{\sqrt{2 \pi a}} e^{-(x-y)^{2} / 2 b} .
$$


Hence when we let $n \rightarrow \infty$ in (3.4), the result is

$$
\begin{aligned}
2 \int_{0}^{s} p(r ; 0,0) d r \int_{0}^{\infty} & g\left(u_{1}, 0, y_{1}\right) \varphi_{1}\left(y_{1}\right) d y_{1} \int_{0}^{\infty} q\left(u_{2}-u_{1} ; y_{1}, y_{2}\right) \varphi_{2}\left(y_{2}\right) d y_{2} . \\
& \cdot \int_{0}^{\infty} q\left(t-r-u_{2} ; y_{2}, y_{3}\right) \varphi_{3}\left(y_{3}\right) d y_{3} .
\end{aligned}
$$

Differentiation with respect to $s$ yields the integrated form of (3.1).

Switching from $\gamma(t)$ to $L^{-}(t)$ and using (2.22), we obtain the conditional law below.

Corollary. For $0<u_{1}<\ldots<u_{m}<r<t$, we have

$$
\begin{gathered}
P\left\{Z\left(u_{1}\right) \in d y_{1} ; \ldots ; Z\left(u_{m}\right) \in d y_{m} ; Z\left(L^{-}(t)\right) \in d y_{m+1} \mid L^{-}(t)=r\right\}= \\
=\sqrt{2 \pi r} g\left(u_{1} ; 0, y_{1}\right) d y_{1} q\left(u_{2}-u_{1} ; y_{1}, y_{2}\right) d y_{2} \ldots q\left(r-u_{m} ; y_{m}, y_{m+1}\right) d y_{m+1} .
\end{gathered}
$$

It is easy to extend Theorem 4 pro forma to a general event belonging to the Borel field generated by the meandering process. We shall use such an extension in the specific case below. For $0<\delta<r$ and $x>0, \xi>0$, we have

$$
\begin{gathered}
P\left\{\max _{\gamma(t)+\delta \leqq u \leqq t} Y(u) \leqq \xi ; Y(i) \in d x \mid L^{-}(t)=r\right\}= \\
=\sqrt{2 \pi r} \int_{0}^{\xi} g(\delta ; 0, y) P^{y}\left\{T_{0}>r-\delta ; \max _{0 \leqq u \leqq r-\delta} Y(u) \leqq \xi ; Y(r-\delta) \in d x\right\} d y .
\end{gathered}
$$

Let us write

$$
M(t)=\max _{0 \leqq s \leqq t} B(s), \quad m(t)=\min _{0 \leqq s \leqq t} B(s) .
$$

For a fixed $\xi>0$ and $0<x \leqq \xi$, we put also

$$
\begin{gathered}
\varphi(t ; y, x) d x=P^{y}\{0<m(t) \leqq M(t) \leqq \xi ; B(t) \in d x\}= \\
\quad=P^{0}\{-y<m(t) \leqq M(t) \leqq \xi-y ; B(t) \in d x-y\} .
\end{gathered}
$$

Observe that the factor $P^{y}\{\ldots\}$ in the right member of (3.6) is then just $\varphi(r-\delta ; y, x)$. It is well known that for $0<y<\xi, 0<x<\xi$, we have

$$
\varphi(t ; y, x)=\sum_{n=-\infty}^{\infty} q(t ; x, y+2 n \xi)
$$

see, e.g., [6; p. 26]. A little inspection shows that for arbitrary $t>0$ and $x>0$, we have

$$
\varphi(t ; 0, x)=\varphi(t ; \xi ; x)=0
$$

and that $\varphi(t ; y, x)$ is periodic in $y$ with period $2 \xi$. The partial derivative of $\varphi$ with respect to $y$ is given formally by

$$
\begin{aligned}
\varphi_{y}(t ; y, x)= & \frac{1}{\sqrt{2 \pi t}} \sum_{n=-\infty}^{\infty}\left\{\frac{x-y-2 n \xi}{t} \exp \left(-\frac{(x-y-2 n \xi)^{2}}{2 t}\right)+\right. \\
& \left.+\frac{x+y+2 n \xi}{t} \exp \left(-\frac{(x+y+2 n \xi)^{2}}{2 t}\right)\right\} .
\end{aligned}
$$


The series converges uniformly in the region $0<a \leqq t \leqq b<\infty, 0 \leqq y \leqq 2 \xi, 0 \leqq x \leqq 2 \xi$ by easy domination. It follows that $\varphi_{y}$ is continuous in $(t, y, x)$ in the region $0<t<\infty, 0 \leqq y \leqq 2 \xi, 0 \leqq x \leqq 2 \xi$ and is indeed represented by the series. In particular, we have

$$
\begin{gathered}
\varphi_{y}(t ; 0, x)=\sqrt{\frac{2}{\pi t}} \sum_{n=-\infty}^{\infty} \frac{x+2 n \xi}{t} \exp \left(-\frac{(x+2 n \xi)^{2}}{2 t}\right)= \\
=2 \sum_{n=0}^{\infty} g(t ; 0,2 n \xi+x)-2 \sum_{n=1}^{\infty} g(t ; 0,2 n \xi-x) .
\end{gathered}
$$

We are now going to evaluate the limit of the right member of (3.6) as $\delta \downarrow 0$. By partial integration, and that $\varphi(r-\delta ; 0, x)=\varphi(r-\delta, \xi, x)=0$, we have

$$
\begin{gathered}
\int_{0}^{\xi} g(\delta ; 0, y) \varphi(r-\delta ; y, x) d y=\frac{1}{\sqrt{2 \pi \delta}} \int_{0}^{\xi} \frac{y}{\delta} e^{-y^{2} / 2 \delta} \varphi(r-\delta ; y, x) d y= \\
=\frac{1}{\sqrt{2 \pi \delta}} \int_{0}^{\xi} e^{-y^{2} / 2 \delta} \varphi_{y}(r-\delta ; y, x) d y
\end{gathered}
$$

As $\delta \nmid 0$, it is easy to see that the last-written integral converges to $(1 / 2) \varphi_{y}(r ; 0, x)$ by the continuity and boundedness of $\varphi_{y}$ mentioned earlier. On the other hand, the left member of (3.6) converges to a similar probability with the $\delta$ there erased. Putting things together, we obtain the next theorem.

Let us put

$$
M^{-}(t)=\max _{\gamma(t) \leqq s \leqq t} Y(s) ; \quad M^{*}(t)=\max _{\gamma(t) \leqq s \leqq \beta(t)} Y(s) .
$$

Thus $M^{-}(t)$ is the maximum of the meandering process ending at $t ; M^{*}(t)$ is the maximum of the excursion process straddling $t$. The latter will be treated in $\S 4$.

Theorem 5. For $0<r<t$ and $0<x<\xi$, we have

$$
\begin{gathered}
P\left\{M^{-}(t) \leqq \xi ; Y(t) \in d x \mid L^{-}(t)=r\right\}= \\
=\sqrt{2 \pi r}\left\{\sum_{n=0}^{\infty} g(r ; 0,2 n \xi+x)-\sum_{n=1}^{\infty} g(r ; 0,2 n \xi-x)\right\} d x .
\end{gathered}
$$

The last expression may be written as

$$
\sum_{n=-\infty}^{\infty} \frac{\partial}{\partial x} \exp \left(-\frac{(2 n \xi+x)^{2}}{2 r}\right) .
$$

Integrating out $d x$ as we may term by term, we obtain

$$
\begin{aligned}
P\left\{M^{-}(t) \leqq \xi \mid L^{-}(t)\right. & =r\}=\sum_{n=-\infty}^{\infty}\left\{\exp \left(-\frac{(2 n)^{2} \xi^{2}}{2 r}\right)-\exp \left(-\frac{(2 n+1)^{2} \xi^{2}}{2 r}\right)\right\}= \\
& =1+2 \sum_{n=1}^{\infty}(-1)^{n} \exp \left(-\frac{n^{2} \xi^{2}}{2 r}\right) .
\end{aligned}
$$


Multiplying (3.12) by (2.22) and integrating over $r$, we have

But

$$
\begin{gathered}
P\left\{M^{-}(t) \leqq \xi ; Y(t) \in d x\right\}= \\
=\int_{0}^{t} 2 p(t-r ; 0,0)\left\{\sum_{n=0}^{\infty} g(r ; 0,2 n \xi+x)-\sum_{n=1}^{\infty} g(r ; 0,2 n \xi-x)\right\} d r .
\end{gathered}
$$

$$
\int_{0}^{t} p(t-r ; 0,0) g(r ; 0, y) d r=p(t ; 0, y)
$$

by the last-exit decomposition (2.11); hence we obtain

$$
\begin{gathered}
P\left\{M^{-}(t) \leqq \xi ; Y(t) \in d x\right\}=2 \sum_{n=0}^{\infty} p(t ; 0,2 n \xi+x)-2 \sum_{n=1}^{\infty} p(t ; 0,2 n \xi-x)= \\
=2 p(t ; 0, x)-\sum_{n=1}^{\infty} q(t ; x, 2 n \xi) .
\end{gathered}
$$

Integrating out $d x$ we get after some simplification

$$
P\left\{M^{-}(t) \leqq \xi\right\}=2 \sum_{n=0}^{\infty}(-1)^{n} P\{n \xi \leqq B(t)<(n+1) \xi\} .
$$

The last formula has also been obtained by John B. Walsh by a direct application of the reflection principle.

We can also derive (3.15) from (3.13) by an interesting detour. The key formula is as follows:

$$
\int_{0}^{t} \frac{1}{\pi \sqrt{r(t-r)}} e^{-x^{2} / 2(t-r)} d r=\sqrt{\frac{2}{\pi t}} \int_{x}^{\infty} e^{-y^{2} / 2 t} d y .
$$

To show this in a probabilistic setting, we rewrite the left member as

$$
\begin{gathered}
2 \int_{0}^{t} \frac{1}{\sqrt{2 \pi r}} d r \int_{x}^{\infty} \frac{y}{\sqrt{2 \pi(t-r)^{3}}} e^{-y / 2(t-r)} d y=2 \int_{0}^{t} p(r ; 0,0) d r \int_{x}^{\infty} g(t-r ; 0, y) d y= \\
=2 \int_{x}^{\infty} d y \int_{0}^{t} p(r ; 0,0) g(t-r ; 0, y) d r
\end{gathered}
$$

and then apply (2.11). to get

$$
2 \int_{x}^{\infty} p(t ; 0, y) d y
$$

which is the right member of (3.16). It follows that if we multiply (3.13) by (2.22) and then integrate with respect to $r$ form 0 to $t$ term by term, the result is

$$
1+4 \sum_{n=1}^{\infty}(-1)^{n} \int_{n \xi}^{\infty} p(t ; 0, y) d y .
$$

This is seen to be the same as the right member of (3.15) by partial summation.

Let us observe that if we put $x=\xi^{2} / 2 r$ in the second member of (3.13), then we obtain the following distribution function:

$$
F(x)=\sum_{n=-\infty}^{\infty}(-1)^{n} e^{-n^{2} x}, \quad 0<x<\infty .
$$


This is known in the theory of theta functions. In fact, using one of Jacobi's identities (see, e.g., [12; Vol. I. p, 8]), we have

$$
F(x)=\prod_{n=1}^{\infty} \frac{1-e^{-n x}}{1+e^{-n x}}, \quad 0<x<\infty .
$$

In this form it becomes clear that $F$ is increasing and $F(0+)=0$; only $F(+\infty)=1$ is obvious from (3.17).

\section{The excursion process and its maximum}

For each $t>0$, the process $Y$ restricted to the interval $(\gamma(t), \beta(t))$ is the excursion process straddling $t$. Thus it is a prolongation of the meandering process ending at $t$ and is defined by

$$
Z(u)=Y(\gamma(t)+u) \text { for } \quad 0 \leqq u \leqq L(t) .
$$

Its fundamental structure is given in the following theorem.

Theorem 6. Let $m \geqq 1,0<u_{1}<\ldots<u_{m}<l$, and $y_{1}, \ldots, y_{m}$ be arbitrary positive numbers. We have for $s+l>t$ :

$$
P\left\{\gamma(t) \in d s ; Z\left(u_{1}\right) \in d y_{1} ; \ldots ; Z\left(u_{m}\right) \in d y_{m} ; L(t) \in d l\right\}=
$$

$$
\begin{gathered}
=2 p(s ; 0,0) d s g\left(u_{1} ; 0, y_{1}\right) d y_{1} q\left(u_{2}-u_{1} ; y_{1}, y_{2}\right) d y_{2} \ldots q\left(u_{m}-u_{m-1} ; y_{m-1}, y_{m}\right) d y_{m} . \\
\cdot g\left(l-u_{m} ; 0, y_{m}\right) d l .
\end{gathered}
$$

Remark. When $m=1$ and when we substitute the random variable $L^{-}(t)$ for the constant $u_{1}$, then (4.1) reduces to (2.19). Such a substitution must of course be justified.

Proof. Again we take $m=2$ and proceed as in the proof of Theorem 4. To lighten the typographical burden we shall write

$$
u_{1}^{\prime}=d_{n k}+u_{1}, \quad u_{2}^{\prime}=d_{n k}+u_{2} .
$$

Let $T_{0}(u)=\inf \{t>u: B(t)=0\}$. Observe that on the set $\left\{u_{2}^{\prime}<\beta(t)\right\}$, we have $\beta(t)=u_{2}^{\prime}+T_{0}\left(u_{2}^{\prime}\right)$. Now we have, in analogy with (3.2),

$$
\begin{gathered}
E\left\{\gamma(t)<s ; \gamma(t)+u_{2}<\beta(t) ; \varphi_{1}\left(Z\left(u_{1}\right)\right) \varphi_{2}\left(Z\left(u_{2}\right)\right) \varphi_{3}(\beta(t))\right\}= \\
=\lim _{n \rightarrow \infty} \sum_{k=1}^{2^{n}} E\left\{\gamma\left(u_{1}^{\prime}\right) \in I_{n k} ; \beta\left(u_{1}^{\prime}\right)>u_{2}^{\prime} ; \varphi_{1}\left(Y\left(u_{1}^{\prime}\right)\right) \varphi_{2}\left(Y\left(u_{2}^{\prime}\right)\right) \varphi_{3}\left(u_{2}^{\prime}+T_{0}\left(u_{2}^{\prime}\right)\right)\right\} .
\end{gathered}
$$

The $k^{\text {th }}$ term in the sum is evaluated by Theorem 1 applied with $t=u_{1}^{\prime}$, followed by Markov property of $Y$ applied successively at $u_{1}^{\prime}$ and $u_{2}^{\prime}$. The result is

$$
\begin{gathered}
2 \int_{I_{n k}} p(r ; 0,0) d r \int_{0}^{\infty} g\left(u_{1}^{\prime}-r ; 0, y_{1}\right) \varphi_{1}\left(y_{1}\right) d y_{1} \int_{0}^{\infty} q\left(u_{2}^{\prime}-u_{1}^{\prime} ; y_{1}, y_{2}\right) \varphi_{2}\left(y_{2}\right) \cdot \\
\cdot E^{y_{2}}\left\{u_{2}^{\prime}+T_{0}>t ; \varphi_{3}\left(u_{2}^{\prime}+T_{0}\right)\right\} d y_{2} .
\end{gathered}
$$


Using (2.1) we see that

$$
E^{y_{2}}\left\{u_{2}^{\prime}+T_{0}>t ; \varphi_{3}\left(u_{2}^{\prime}+T_{0}\right)\right\}=\int_{\left(t-u_{2}^{\prime}\right)}^{\infty} g\left(v-u_{2}^{\prime} ; 0, y_{2}\right) \varphi_{3}\left(u_{2}^{\prime}+v\right) d v
$$

When $r \in I_{n k}, r_{n}=d_{n k}$, so that $u_{1}^{\prime}=r_{n}+u_{1}, u_{2}^{\prime}=r_{n}+u_{2}$. Substituting into (4.2), we see that the sum there is equal to

$$
\begin{gathered}
2 \int_{0}^{s} p(r ; 0,0) d r \int_{0}^{\infty} g\left(r_{n}-r+u_{1} ; 0, y_{1}\right) \varphi_{1}\left(y_{1}\right) d y_{1} \int_{0}^{\infty} q\left(u_{2}-u_{1} ; y_{1}, y_{2}\right) \varphi_{2}\left(y_{2}\right) d y_{2} \cdot \\
\cdot \int_{\left(1-u_{2}^{\prime}\right)^{+}}^{\infty} g\left(v ; 0, y_{2}\right) \varphi_{3}\left(u_{2}^{\prime}+v\right) d v
\end{gathered}
$$

Letting $n \rightarrow \infty$ so that $r_{n} \rightarrow r$, we see that the result is tentamount to

$$
\begin{gathered}
P\left\{\gamma(t) \in d s ; \gamma(t)+u_{2}<\beta(t) ; Z\left(u_{1}\right) \in d y_{1} ; Z\left(u_{2}\right) \in d y_{2} ; \beta(t)-\gamma(t)-u_{2} \in d v\right\}= \\
=2 p(s ; 0,0) d s g\left(u_{1}, 0, y_{1}\right) d y_{1} q\left(u_{2}-u_{1} ; y_{1}, y_{2}\right) d y_{2} g\left(v ; 0, y_{2}\right) d v,
\end{gathered}
$$

if $s+u_{2}+v>t ;=0$ otherwise. Writing $l$ for $u_{2}+v$ we see that this is (4.1) when $m=2$.

Corollary. We have for $0<s<t ; 0<u_{1}<\ldots<u_{m}<l$, and arbitrary positive $y_{1}, \ldots, y_{m}$ :

$$
\begin{gathered}
P\left\{Z\left(u_{1}\right) \in d y_{1} ; \ldots ; Z\left(u_{m}\right) \in d y_{m} \mid \gamma(t)=s, L(t)=l\right\}= \\
=\sqrt{8 \pi l^{3}} g\left(u_{1} ; 0, y_{1}\right) d y_{1} q\left(u_{2}-u_{1} ; y_{1}, y_{2}\right) d y_{2} \ldots q\left(u_{m}-u_{m-1} ; y_{m-1}, y_{m}\right) \cdot \\
\cdot g\left(l-u_{m} ; 0, y_{m}\right) d y_{m} .
\end{gathered}
$$

Proof. Rewrite $(2,20)$ as

$$
P\{\gamma(t) \in d s ; L(t) \in d l\}=\frac{d s d l}{2 \pi \sqrt{s l^{3}}} \text { for } t-l<s<t
$$

and divide (4.1) by (4.4). The result is (4.3).

Note that the factor $\sqrt{8 \pi l^{3}}$ in (4.3) is just $1 / \theta(l)$; so that if we put $m=1$ there and integrate out $d y_{1}$, the result indeed checks with Proposition 2 .

We can now use the method of finding the distribution of $M^{-}(t)$ in Theorem 5 to find that of $M^{*}(t)$, by basing it on Theorem 6 instead of Theorem 4 .

Theorem 7. For $0<t-s<l<\infty$, we have

$$
P\left\{M^{*}(t) \leqq \xi \mid \gamma(t)=s, L(t)=l\right\}=1+2 \sum_{n=1}^{\infty}\left(1-\frac{4 n^{2} \xi^{2}}{l}\right) \exp \left(-\frac{2 n^{2} \xi^{2}}{l}\right) .
$$

Proof. It follows from the Corollary to Theorem 6 that for sufficiently small positive $\delta$ and $\varepsilon$, we have

$$
\begin{gathered}
P\left\{\max _{s+\delta \leqq u \leqq s+l-\varepsilon} Y(u) \leqq \xi \mid \gamma(t)=s, L(t)=l\right\}= \\
=\sqrt{8 \pi l^{3}} \int_{0}^{\xi} g(\delta ; 0, y) d y \int_{x=0}^{\xi} P^{y}\left\{T_{0}>l-\delta-\varepsilon ; \max _{0 \leqq u \leqq l-\delta-\varepsilon} Y(u) \leqq\right. \\
\leqq \xi ; Y(l-\delta-\varepsilon) \in d x\} g(\varepsilon ; 0, x) .
\end{gathered}
$$


Using the function $\varphi$ in (3.7), the second integral above may be written as

$$
\int_{0}^{\xi} \varphi(l-\delta-\varepsilon ; y, x) g(\varepsilon ; 0, x) d x .
$$

By the argument in (3.11), we see that as $\varepsilon \downarrow 0$ this integral converges to $2^{-1} \varphi_{x}(l-\delta ; y, 0)$ where $\varphi_{x}$ is the partial derivative of $\varphi(t ; y, x)$ with respect to $x$. If we substitute this limit in (4.6), its right member becomes

$$
\sqrt{8 \pi l^{3}} \frac{1}{2} \int_{0}^{\xi} g(\delta ; 0, y) \varphi_{x}(l-\delta ; y, 0) d y .
$$

The same argument shows that as $\delta \downarrow 0$, this integral converges to

$$
\sqrt{8 \pi l^{3}} \cdot \frac{1}{4} \varphi_{x y}(l ; 0,0) .
$$

Here $\varphi_{x y}(t ; y, 0)$ is the partial derivative of $\varphi_{x}(t ; y, 0)$ with respect to $y$. Its continuity and boundedness must be checked as done before for $\varphi_{y}$ in (3.9). From (3.10) with $x$ and $y$ interchanged we obtain by differentiation

$$
\varphi_{x y}(t ; y, 0)=\sqrt{\frac{2}{\pi t^{3}}} \sum_{n=-\infty}^{\infty}\left(1-\frac{(y+2 n \xi)^{2}}{t}\right) \exp \left(-\frac{(y+2 n \xi)^{2}}{2 t}\right) .
$$

Hence the expression in (4.8) is equal to the right member of (4.5) as asserted. Theorem 8 is proved.

Putting $x=2 \xi^{2} / l$ in the right member of (4.5), we see that the function $F$ below,

$$
F(x)=1+2 \sum_{n=1}^{\infty} e^{-n^{2} x}\left(1-2 n^{2} x\right), \quad 0<x<\infty,
$$

is a distribution function. Observe that $F(0+)=0$ as a consequence of Theorem 7 , but this cannot be deduced by putting $x=0$ in (4.9) because the series does not converge uniformly in the neighborhood of $x=0$. Direct analytical verification of the properties $F$ is not trivial.* One method is to pass to Laplace transforms, and using Euler's partial fraction expansion of $\left(e^{z}-1\right)^{-1}$ to get

$$
\hat{F}(\lambda)=\int_{0}^{\infty} e^{-\lambda x} d F(x)=\frac{4 \pi^{2} \lambda e^{-2 \pi \sqrt{\lambda}}}{\left(1-e^{-2 \pi \sqrt{\lambda}}\right)^{2}} .
$$

This shows $F(0+)=\lim _{\lambda \rightarrow \infty} \lambda \hat{F}(\lambda)=0$. But to recognize the last member as the Laplace transform of a distribution function, we need the formula, not so well known but computable:

$$
E\left(e^{-\lambda s}\right)=\frac{2 \pi \sqrt{\lambda} e^{-\pi \sqrt{\lambda}}}{1-e^{-2 \pi \sqrt{\lambda}}}
$$

\footnotetext{
* In fact, when the distribution was first found, the only confirmation was obtained by Iglehart
} on the computer. 
where $S$ is the first entrance time of the 3-dimensional Bessel process into the singleton $\{\pi / \sqrt{2}\}$. Thus $F$ is the distribution of the sum of two independent copies of $S$. For a deduction which apparently goes in the opposite direction, see [13].

A quicker analytical verification of the properties of $F$ has been furnished by W. A. Veech as follows. Introduce the theta function

We have then

$$
\vartheta(x)=1+2 \sum_{n=1}^{\infty} e^{-n^{2} \pi x} .
$$

$$
F(x)=\vartheta(x)+2 x \vartheta^{\prime}(x)
$$

Using Jacobi's functional equation

we obtain

$$
\vartheta(x)=\frac{1}{\sqrt{x}} \vartheta\left(\frac{1}{x}\right)
$$

$$
F(\pi x)=-\frac{2}{\sqrt{x^{3}}} \vartheta^{\prime}\left(\frac{1}{x}\right)=\frac{4 \pi}{\sqrt{x^{3}}} \sum_{n=1}^{\infty} n^{2} e^{-n^{2} \pi / x} .
$$

If we put $\pi / x=z$, we have

$$
F\left(\frac{\pi^{2}}{z}\right)=\frac{4}{\sqrt{\pi}} z^{3 / 2} \sum_{n=1}^{\infty} n^{2} e^{-n^{2} z}
$$

and it is now clear that $\lim _{x \downarrow 0} F(x)=0$. It is trivial from $(4.9)$ that $\lim _{x \rightarrow \infty} F(x)=1$.

Next we get from (4.9) that

$$
F^{\prime}(x)=2 \sum_{n=1}^{\infty} e^{-n^{2} x} n^{2}\left(2 n^{2} x-3\right) .
$$

Hence $F^{\prime}(x)>0$ for $x>3 / 2$. On the other hand, we get from (4.10) that

$$
\pi F^{\prime}(\pi x)=\frac{4 \pi}{\sqrt{x^{7}}} \sum_{n=1}^{\infty} e^{-n^{2} \pi / x} n^{2}\left(n^{2} \pi-\frac{3 x}{2}\right) .
$$

Hence $F^{\prime}(x)>0$ for $0<x<2 \pi^{2} / 3$. Since $3 / 2<2 \pi^{2} / 3$, we have shown that $F^{\prime}(x)>0$ for all $x>0$.

Iglehart [7] had the idea of studying a "scaled meandering" by the methods of weak convergence, and this was extended to a "scaled excursion" by W. D. Kaigh [9]. In the terminology used here, "scaled" means "of duration equal to one (unit)". Kaigh's result corresponding to Theorem 7 reads as follows (private communication). Let $\left\{X_{n}, n \geqq 1\right\}$ be independent, identically distributed random variables such that $X_{1}$ takes the values +1 and -1 with probability $1 / 2$ each; and let $S_{n}=\sum_{k=1}^{n} X_{k}$; $T=\min \left\{n \geqq 1 \mid S_{n}=0\right\}$. Then

$$
\lim _{n \rightarrow \infty} P\left\{\max _{1 \leqq k \leqq 2 n}\left|S_{k}\right| / \sqrt{2 n} \leqq x \mid T=2 n\right\}=F\left(2 x^{2}\right)
$$


where $F$ is given in (4.9). A result corresponding to (3.13) is obtained when the condition " $T=2 n$ " above is replaced by " $T>2 n$ ". These results can then be shown to hold for more general $X_{n}$ 's by an invariance principle due to Iglehart, and yield schemes which converge weakly to the scaled meandering and excursion processes. Further investigation of the various relations will appear in a paper by Durrett and Iglehart [5].

\section{A curious connection}

There is a way of reaching Theorem 7 via Theorem 5 which involves some interesting calculations.

Proposition 8. For $0<x<\xi$ and $u>0$ we have

$$
P^{x}\left\{T_{0}<T_{\xi} ; T_{0} \in d u\right\}=-\sum_{n=-\infty}^{\infty} p_{x}(u ; 0,2 n \xi+x) d u
$$

where $p_{x}(u, x, y)=(\partial / \partial x) p(u ; x, y)$.

Proof. The probability in the left member of (5.1) is expressible as

$$
\begin{gathered}
\lim _{\varepsilon \downarrow 0} \int_{y=0}^{\xi} P^{x}\left\{T_{0}>\right. \\
\left.u-\varepsilon ; \max _{0 \leqq s \leqq u-\varepsilon} Y(s) \leqq \xi ; Y(u-\varepsilon) \in d y\right\} P^{y}\left\{T_{0} \in d \varepsilon\right\}= \\
=\lim _{\varepsilon \downarrow 0} \int_{0}^{\xi} \varphi(u-\varepsilon ; x, y) g(\varepsilon ; 0, y) d y
\end{gathered}
$$

in the notation of (3.7). The last limit was evaluated under (4.7) with $u=l-\delta$ and $x$ and $y$ interchanged as $2^{-1} \varphi_{y}(u ; x, 0)$, which is seen to equal the right member of $(5.1)$.

The Laplace transform $E^{x}\left\{T_{0}<T_{\xi} ; e^{-\lambda T_{0}}\right\}$ is known (see. e.g., [8; p. 29]) and (5.1) may be obtained by inverting it. But the argument above, part of the proof of Theorem 7, is more in the spirit of this paper.

Now we multiply (3.12) with (5.1), and observe that the right member of (3.12) is just

We obtain thus

$$
-\sqrt{2 \pi r} \sum_{n=-\infty}^{\infty} p_{x}(r ; 0,2 n \xi+x) .
$$

$$
\begin{aligned}
& \int_{x=0}^{\xi} P\left\{M^{-}(t) \leqq \xi ; Y(t) \in d x \mid L^{-}(t)=r\right\} P^{x}\left\{T_{0}<T_{\xi} ; T_{0} \in d u\right\}= \\
& =\sqrt{2 \pi r} \int_{0}^{\xi} \sum_{n=-\infty}^{\infty} p_{x}(r ; 0,2 n \xi+x) \sum_{n=-\infty}^{\infty} p_{x}(u ; 0,2 n \xi+x) d x .
\end{aligned}
$$

If we put $M^{+}(t)=\max _{t \leqq s \leqq \beta(t)} Y(s)$, then it is clear that

$$
P\left\{M^{+}(t) \leqq \xi ; \beta(t) \in t+d u \mid Y(t)=x\right\}=P^{x}\left\{T_{0}<T_{\xi} ; T_{0} \in d u\right\} .
$$

Using this in (5.2) we see that its left member is just

$$
P\left\{M^{*}(t) \leqq \xi ; L(t)=r+d u \mid L^{-}(t)=r\right\} .
$$


Dividing this by (2.23), we get

$$
\begin{gathered}
P\left\{M^{*}(t) \leqq \xi \mid L^{-}(t)=r, L(t)=r+u\right\}= \\
=\sqrt{8 \pi(r+u)^{3}} \int_{0}^{\xi} \sum_{m=-\infty}^{\infty} \sum_{n=-\infty}^{\infty} p_{x}(r ; 0,2 m \xi+x) p_{x}(u ; 0,2 n \xi+x) d x .
\end{gathered}
$$

It is remarkable that the last-written series can be evaluated (term by term). Using the fact that $p$ satisfies the heat equation

$$
\frac{1}{2} \frac{\partial}{\partial t} p(t ; x, y)=\frac{\partial^{2}}{\partial x^{2}} p(t ; x, y)
$$

we transform the integral by partial integration as follows:

$$
\begin{gathered}
\sum_{m} \sum_{n}\left\{p(r ; 0,(2 m+1) \xi) p_{x}(u ; 0,(2 n+1) \xi)-p(r ; 0,2 m \xi) p_{x}(u ; 0,2 n \xi)\right\}- \\
-2 \sum_{m} \sum_{n} \frac{\partial}{\partial u} \int_{0}^{\xi} p(r ; 0,2 m \xi+x) p(u ; 0,2 n \xi+x) d x
\end{gathered}
$$

where all the sums range over all integers. The first double sum above vanishes because $p_{x}(u ; 0, y)$ is an odd function of $y$. The second may be evaluated as a repeated integral by putting $k=m-n$ and summing first over $n$. Making use of the convolution property of $p$ as well as its being a function of $(x-y)^{2}$, we reduce the sum to

$$
-2 \frac{\partial}{\partial u}\left\{\frac{1}{2} p(r+u ; 0,0)+\sum_{k=1}^{\infty} p(r+u ; 0,2 k \xi)\right\} .
$$

Carrying out the partial differentiation and substituting in (5.3), we see that the right member of (5.3) agrees with that of (4.5) with $l=r+u$ and $s=t-r$. This was indeed the way formula (4.5) was first "computed out". It is recorded here as an item of curiosity.

\section{Occupation times}

As another application of Theorem 6, we can calculate easily the expected occupation time during a meandering or excursion. Let $(a, b) \subset(0, \infty)$, and define

$$
\begin{aligned}
S^{-}(t ;(a, b)) & =\int_{\gamma(t)}^{t} I_{(a, b)}(Y(u)) d u \\
S(t ;(a, b)) & =\int_{\gamma(t)}^{\beta(t)} I_{(a, b)}(Y(u)) d u ;
\end{aligned}
$$

where $I_{(a, b)}$ is the indicator of $(a, b)$. We begin with the observation that for $0<t$, $x>0, y>0$, we have

$$
\int_{0}^{t} g(s ; 0, x) g(t-s ; 0, y) d s=g(t ; 0, x+y) .
$$


This follows at once from (2.1) from the meaning of $g$ as density of first entrance time and basic properties of the Brownian motion. Now we have by (4.3)

$$
\begin{aligned}
E\{S(t ; d x) \mid \gamma(t)=s, L(t)=l\} & =\int_{0}^{l} P\{Z(u) \in d x \mid \gamma(t)=s, L(t)=l\} d s \\
& =\int_{0}^{l} \sqrt{8 \pi l^{3}} g(u ; 0, x) g(l-u ; 0, x) d u d x \\
& =\sqrt{8 \pi l^{3}} g(l ; 0,2 x) d x=4 x e^{-2 x^{2} / l} d x .
\end{aligned}
$$

This result is due to Lévy (cf. his derivation on p. 221 of [11]). It constitutes the basis of his fundamental theorem on local time cited at the end of this section.

To obtain the unconditioned expected occupation time we multiply (6.2) by (2.20) with $l=u-s$, and integrate over $s$ :

$$
E\{S(t ; d x)\} / d x=\frac{1}{2 \pi} \int_{0}^{t} \frac{d s}{\sqrt{s}} \int_{t-s}^{\infty} \frac{4 x}{\sqrt{l^{3}}} e^{-2 x^{2} / l} d l .
$$

Setting $y=2 x \sqrt{(t-s) / l}$ we obtain

$$
\frac{1}{x \sqrt{t-s}} \int_{0}^{2 x} e^{-y^{2} / 2(t-s)} d y
$$

for the second integral in (6.3), so that

$$
E\{S(t ; d x)\} / d x=\frac{2}{\pi} \int_{0}^{t} \frac{d s}{\sqrt{s(t-s)}} \int_{0}^{2 x} e^{-y^{2} / 2(t-s)} d y .
$$

This can be evaluated by (3.16). More directly, we cast it into a probabilistic form, using (2.6) and (2.7) after integrating the latter over $(y, \infty)$,

$$
\begin{gathered}
2 \int_{0}^{t} P\{\gamma(t) \in d s\} \int_{0}^{2 x} P\{Y(t)>y \mid \gamma(t)=s\} d y= \\
\quad=2 \int_{0}^{2 x} P\{Y(t)>y\} d y=2 E\{Y(t) \wedge 2 x\} .
\end{gathered}
$$

Next, we calculate the expected occupation time during a meandering. We have by (3.5),

$$
\begin{aligned}
E\left\{S^{-}(t ; d x) / d x \mid L^{-}(t)=r\right\} & =\int_{0}^{r} P\left\{Z(u) \in d x \mid L^{-}(t)=r\right\} d u \\
& =\int_{0}^{r} \sqrt{2 \pi r} g(u ; 0, x) P^{x}\left\{T_{0}>r-u\right\} d u \\
& =\sqrt{2 \pi r} \int_{0}^{r} P^{0}\left\{T_{x} \in d u\right\} P^{x}\left\{T_{2 x}>r-u\right) d u \\
& =\sqrt{2 \pi r} P^{0}\left\{T_{x}<r<T_{2 x}\right\} .
\end{aligned}
$$

Recall the notation $M(r)=\max _{0 \leqq s \leqq r} B(s)$ and the basic formula

$$
P\left\{T_{x}<r\right\}=P\{M(r)>x\}=\sqrt{\frac{2}{\pi r}} \int_{x}^{\infty} e^{-y^{2} / 2 r} d y .
$$


Using this in the last member of (6.6), we obtain

$$
E\left\{S^{-}(t ; d x) / d x \mid L^{-}(t)=r\right\}=2 \int_{x}^{2 x} e^{-y^{2} / 2 r} d y
$$

Hence by (2.22),

$$
E\left\{S^{-}(t ; d x)\right\} / d x=\int_{0}^{t} \frac{2 d r}{\pi \sqrt{r(t-r)}} \int_{x}^{2 x} e^{-y^{2} / 2 r} d y .
$$

Comparison with (6.4) and (6.5) shows that this is equal to

$$
2 E\{Y(t) \wedge 2 x\}-2 E\{Y(t) \wedge x\} .
$$

For (6.9) it is perhaps easier to calculate the difference below:

$$
\begin{aligned}
E\{S(t ; d x)\} / d x-E\left\{S^{-}(t ; d x)\right\} / d x & =E\left\{\int_{t}^{\beta(t)} I_{(d x)}(Y(u)) d u\right\} / d x \\
& =\int_{0}^{\infty} 2 p(t ; 0, y) d y \int_{0}^{\infty} q(u ; y, x) d u \\
& =\int_{0}^{\infty} 2 p(t ; 0, y) 2(x \wedge y) d y . \\
& =2 E\{Y(t) \wedge x\} .
\end{aligned}
$$

Subtracting this from (6.5) we get (6.9).

Moments of higher order can be calculated in the same manner. For instance, we have

since

$$
E\left\{S(t ;(a, b))^{2} \mid \gamma(t)=s, L(t)=l\right\}=
$$$$
=2 \int_{0}^{l} d u_{1} \int_{0}^{l-u_{1}} d u_{2} \int_{a}^{b} d x_{1} \int_{a}^{b} d x_{2} P\left\{Z\left(u_{1}\right) \in d x_{1} ; Z\left(u_{1}+u_{2}\right) \in d x_{2} \mid \gamma(t)=s, L(t)=l\right\}=
$$$$
=2 \sqrt{8 \pi l^{3}} \int_{a}^{b} d x_{1} \int_{a}^{b} d x_{2} \int_{0}^{l} d u_{1} \int_{0}^{l-u_{1}} d u_{2} g\left(u_{1} ; 0, x_{1}\right) \text {. }
$$$$
\cdot \int_{\left|x_{1}-x_{2}\right|}^{x_{1}+x_{2}} g\left(u_{2} ; 0, z\right) d z g\left(l-u_{1}-u_{2} ; 0, x_{2}\right)
$$

$$
q\left(u ; x_{1}, x_{2}\right)=\int_{\left|x_{1}-x_{2}\right|}^{x_{1}+x_{2}} g(u ; 0, z) d z .
$$

By (6.1) this simplifies to

$$
\begin{gathered}
2 \sqrt{8 \pi l^{3}} \int_{a}^{b} d x_{1} \int_{a}^{b} d x_{2} \int_{\left|x_{1}-x_{2}\right|}^{x_{1}+x_{2}} g\left(l ; 0, x_{1}+x_{2}+z\right) d z= \\
=4 \int_{a}^{b} d x_{1} \int_{a}^{b} d x_{2} \int_{\left|x_{1}-x_{2}\right|}^{x_{1}+x_{2}}\left(x_{1}+x_{2}+z\right) \exp \left(-\left(x_{1}+x_{2}+z\right)^{2} / 2 l\right) d z .
\end{gathered}
$$

It is possible, but perhaps futile, to evaluate this in exact terms.

In general, we have for integer $k \geqq 2$ :

$$
E\left\{S(t ;(a, b))^{k} \mid \gamma(t)=s, L(t)=l\right\}=
$$

$$
\begin{gathered}
=2 k ! \int_{a}^{b} d x_{1} \ldots \int_{a}^{b} d x_{k} \int_{\left|x_{1}-x_{2}\right|}^{x_{1}+x_{2}} d z_{1} \ldots \int_{\left|x_{k-1}-x_{k}\right|}^{x_{k-1}+x_{k}} d z_{k-1}\left(x_{1}+z_{1}+\ldots+z_{k-1}+x_{k}\right) \\
\exp \left\{-\frac{\left(x_{1}+z_{1}+\ldots+z_{k-1}+x_{k}\right)^{2}}{2 l}\right\} .
\end{gathered}
$$


This remains true for $k=1$ by (6.2) when there is no $z$ in the formula. A little inspection shows that

$$
\int_{\left|x_{1}-x_{2}\right|}^{x_{1}+x_{2}} d z_{1} \ldots \int_{\left|x_{k-1}-x_{k}\right|}^{x_{k-1}+x_{k}} d z_{k-1}\left(x_{1}+z_{1}+\ldots+z_{k-1}+x_{k}\right) \leqq(k+1) 2^{k-1} x_{1} \ldots x_{k} .
$$

Using this in (6.10) we obtain the inequality below.

Theorem 9. For any $s>0, l>0,0<a<b<\infty$ and integer $k \geqq 1$, we have

$$
E\left\{S(t ;(a, b))^{k} \mid \gamma(t)=s, L(t)=l\right\} \leqq(k+1) !\left(b^{2}-a^{2}\right)^{k} .
$$

It is remarkable that the estimate does not depend on $s$ or $l$. In particular, if $a=0, b=\varepsilon>0$, we get

$$
E\left\{S(t ;(0, \varepsilon))^{k}\right\} \leqq(k+1) ! \varepsilon^{2 k}
$$

Consequently we have for any $\lambda<1$

$$
E\left\{\exp \left(\frac{\lambda S(t ;(0, \varepsilon))}{\varepsilon^{2}}\right)\right\}<\infty .
$$

On pp. 338-9 of [10], Lévy asserted an asymptotic form of (6.12) for $k=2$ (with some constant in lieu of 3 ! in front of $\varepsilon^{4}$ there), "par raison d'homogénéité". This is not clear to me. It is true that for the unconstrained Brownian motion, starting from any $x>0$, the occupation time in $(0, \varepsilon)$ until the first entrance into zero has a finite second moment. But to transport such a result to an arbitrary excursion seems to require an additional argument. This is now supplied and generalized in Theorem 9. Lévy's estimate played an essential role of the proof of his fundamental result that

$$
\lim _{\varepsilon \nmid 0} \frac{1}{2 \varepsilon} \text { measure }\{s \mid s \leqq t ; B(t) \in(-\varepsilon, \varepsilon)\}
$$

exists almost surely for every $t>0$, and equals the local time at zero up to time $t$. As far as I can ascertain, no other author has returned to his original approach (see the remarks on p. 44 of [8]). For a new derivation of a related result about "downcrossings", see [4b].

It should be possible to compute from (6.10) the exact value of, say,

$$
\lim _{\varepsilon \downarrow 0} E\left\{\frac{1}{\varepsilon^{k}} S(t ;(a, a+\varepsilon))^{k} \mid \gamma(t)=s, L(t)=l\right\},
$$

and thereby to determine the limit distribution of $S(t ;(a, a+\varepsilon)) / \varepsilon$ as $\varepsilon \downarrow 0$. The latter exists because of obvious tightness and Carleman's condition by (6.12). What it is remains to be seen. 


\section{Analogy with boundary theory for Markov chains}

We may follow the recipe given on pp. 153-154 of [2] or pp. 85-86 of [3] to derive the basic quantities for the excursion process. First, here is the preliminary list of correspondences:

\begin{tabular}{c|c|c|c|c|c}
$\mathrm{MC}$ & $a$ & $p_{i j}(t)$ & $f_{i j}(t)^{*}$ & $l_{i}(t)$ & $e_{i}^{a}$ \\
\hline $\mathrm{BM}$ & 0 & $p(t ; x, y) d y$ & $q(t ; x, y) d y$ & $g(t ; 0, x) d x$ & $1 d x$
\end{tabular}

Apart from notations, these are obvious except possibly the last item. Now since the Brownian motion is spatially homogeneous, the Borel-Lebesgue measure on the line is invariant with respect to its transition semigroup, i.e.,

$$
\int_{-\infty}^{\infty} 1 d x p(t ; x, y) d y=1 d y .
$$

Hence $1 d x$ plays the role of $e_{i}^{a}$ (see p. 68 of [3]). Next, we compute the quantity corresponding to

which is

$$
e_{j}^{a}-\sum_{i} e_{i}^{a} f_{i j}(t)
$$

$$
d y-\int_{0}^{\infty} d x q(t ; x, y) d y=d y-P^{y}\left\{T_{0}>t\right\} d y=P^{y}\left\{T_{0} \leqq t\right\} d y .
$$

According to the recipe the entrance law $\left\{\eta_{j}^{a}(t)\right\}$ with respect to the minimal semigroup $\{\Phi(t)\}$ is then obtained as follows:

$$
\eta_{j}^{a}(t)=\frac{d}{d t}\left\{e_{j}^{a}-\sum_{i} e_{t} f_{i j}(t)\right\}
$$

Here the corresponding step yields

$$
\frac{d}{d t} P^{y}\left\{T_{0} \leqq t\right\} d y=g(t ; 0, y) d y .
$$

Thus $g$ is indeed the entrance law to the excursion process. Next, the formula

becomes

$$
\sigma^{a a}(t)=\sum_{i} \eta_{i}^{a}(t) L_{i}^{a}(\infty)
$$

$$
\sigma(t)=\int_{0}^{\infty} g(t ; 0, x) 1 d x=\frac{1}{\sqrt{2 \pi t}}
$$

\footnotetext{
* This is the transition probability for the minimal process, not the first entrance time density.
} 
as computed in (2.12). The fundamental integral equation

$$
1=\int_{0}^{t} E^{a}(d s) \sigma^{a a}(t-s), \quad 0<t<\infty ;
$$

becomes

$$
1=\int_{0}^{t} E(d s) \frac{1}{\sqrt{2 \pi(t-s)}}, \quad 0<t<\infty
$$

from which we obtain the unique solution

$$
e(s) d s=E(d s)=\sqrt{\frac{2}{\pi s}} d s .
$$

This is just $2 p(s ; 0,0) d s$, the probability density at zero for $Y(s)$. From a regenerative point of view this would be the fundamental quantity. Note that (7.2) turns out to be the famous arc sin law (cf. (2.6))

$$
1=\int_{0}^{t} \frac{1}{\pi \sqrt{s(t-s)}} d s=\frac{2}{\pi} \arcsin 1, \quad 0<t<\infty,
$$

and that there is a kind of reciprocity here: $e(t)=2 \sigma(t)$. Next, from the recipe

we get from (7.1)

$$
\sigma(t)=\int_{t}^{\infty} \theta(s) d s
$$

$$
\theta(t)=-\frac{d}{d t} \sigma(t)=\frac{1}{\sqrt{8 \pi t^{3}}}
$$

as given in (2.18). After these identifications further analogy with boundary theory may be pursued easily. See for instance [4a] which contains the generic form of (2.20) above.

\section{References}

1. Chung, K. L., Markov Chains with Stationary Transition Probabilities, second edition, SpringerVerlag 1967.

2. Chung, K. L., On the boundary theory for Markov chains II, Acta Math. 115 (1966), 111-163.

3. Chung, K. L., Lectures on Boundary Theory for Markov Chains, Princeton University Press 1970.

4. Chung, K. L., Maxima in Brownian excursions, Bull. Amer. Math. Soc. 81 (1975), 742-745.

4a. Chung, K. L., A bivariate distribution in regeneration. J. Appl. Prob. 12 (1975), 837-839.

4b. Chung, K. L. and Durretr, R., Downcrossings and local time. To appear in Zeitschrift für Wahrscheinlichkeitstheorie.

5. Durrett, R. T. and Iglehart, D. L., Functionals of Brownian meandering and Brownian excursion. To appear.

6. Freedman, D., Brownian Motion and Diffusion, Holden-Day 1971.

7. IglehaRT, D. L., Functional central limit theorems for random walks conditioned to stay positive, Ann. Probability 2 (1974), 608-619.

8. Ito, K. and McKean, Jr. H. P., Diffusion Processes and Their Sample Paths, Springer-Verlag, 1965. 
9. KAIGH, W. D., An invariance principle for random walk conditioned by a late return to zero. To appear in Ann. of Probability.

10. LÉvy, Paul, Sur certains processus stochastiques homogènes, Compositio Math. 7 (1939), 283-339.

11. LÉvY, PAUL, Processus stochastiques et mouvement brownien, second edition, Gauthier-Villars 1965 (first ed. 1948).

12. Pólya, G. and SzEgö, G., Aufgaben der Lehrsätze aus der Analysis, Springer-Verlag 1925. 13. Williams, D., The Ito excursion law for Brownian motion. To appear.

Received January 7, 1976

\section{Added in proof}

Since the manuscript was prepared more than a year ago, I have given extensive lectures on it especially in Peking and Amsterdam (summer and fall of 1975), during the course of which the following amendments were made.

(1) Mr. Berber gave a quicker proof of Theorem 1 by using the equivalent Brownian motion $t B(1 / t)$, thereby reducing the consideration of $(\gamma(t), Y(t))$ to $(Y(t), \beta(t))$.

(2) Besides [5], the following two papers also treat the distributions of the maxima:

D. P. Kennedy, The distribution of the maximum Brownian excursion (to appear in J. Appl. Probability).

D. R. Miller, The distributions of the suprema of the Brownian paths (to appear).

The distribution in (4.9) was obtained by N. H. Kuiper in "Tests concerning random points on a circle". Indag. Math. 22 (1960), 32-37; 38-47. There it appeared as the distribution of the maximum minus the minimum in a Brownian bridge.

(3) Here is an unexpected result. If we denote the distribution in (4.9) by $F_{2}$ and that in (3.17) by $F_{1}$, then we have $F_{2}=F_{1} * F_{1}$ where $*$ denotes convolution. This is easily verified by Laplace transform and made my reference to $E\left(e^{-\lambda S}\right)$ on pp. 168-169 unnecessary. The curious coincidence is still unexplained, as well as its relation to previously known results by Ito-McKean and D. Williams, concerning the path decomposition of an excursion into two Bessel (3) motions pieced together back-to-back, see $\S 2.10$ of [8].

(4) I have calculated the first four moments of the unknown limit distribution mentioned after (6.13), namely that of $S(t ;(0, \varepsilon)) / \varepsilon^{2}$ as $\varepsilon \downarrow 0$. They are: $2,16 / 3$, $17 \times 2^{4} / 15,31 \times 2^{8} / 105$. The corresponding central moments are: $0,4 / 3,32 / 15$, $69 \times 16 / 105$.

Kai Lai Chung

Department of Mathematics

Stanford University

Stanford, California 94305

U.S.A. 